\title{
Analyzing Large Sparse Markov Chains of Kronecker Products
}

\author{
Tuğrul Dayar \\ Department of Computer Engineering \\ Bilkent University \\ TR-06800 Bilkent, Ankara, Turkey \\ Email: tugrul@cs.bilkent.edu.tr
}

\begin{abstract}
Kronecker products are used to define the underlying Markov chain (MC) in various modeling formalisms, including compositional Markovian models, hierarchical Markovian models, and stochastic process algebras. The motivation behind using a Kronecker structured representation rather than a flat one is to alleviate the storage requirements associated with the MC. With this approach, systems that are an order of magnitude larger can be analyzed on the same platform.
\end{abstract}

In the Kronecker based approach, the generator matrix underlying the $\mathrm{MC}$ is represented using Kronecker products [6] of smaller matrices and is never explicitly generated. The implementation of transient and steady-state solvers rests on this compact Kronecker representation, thanks to the existence of an efficient vector-Kronecker product multiplication algorithm known as the shuffle algorithm [6]. The transient distribution can be computed through uniformization using vector-Kronecker product multiplications. The steady-state distribution also needs to be computed using vector-Kronecker product multiplications, since direct methods based on complete factorizations, such as Gaussian elimination, normally introduce new nonzeros which cannot be accommodated. The two papers [2], [10] provide good overviews of iterative solution techniques for the analysis of MCs based on Kronecker products. Issues related to reachability analysis, vector-Kronecker product multiplication, hierarchical state space generation in Kronecker based matrix representations for large Markov models are surveyed in [5].

Throughout our discussion, we make the assumption that the MC at hand does not have unreachable states, meaning it is irreducible. And we take an algebraic view [7] to discuss recent results related to the analysis of MCs based on Kronecker products independently from modeling formalisms. We provide background material on the Kronecker representation of the generator matrix underlying a CTMC, show that it has a rich structure which is nested and recursive, and introduce a small CTMC whose generator matrix is expressed as a sum of Kronecker products; this CTMC is used as a running example throughout the discussion. We also consider preprocessing of the Kronecker representation so as to expedite numerical analysis. We discuss permuting the nonzero structure of the underlying CTMC symmetrically by reordering, changing the orders of the nested blocks by grouping, and reducing the size of the state space by lumping. The steady-state analysis of CTMCs based on Kronecker products is discussed for block iterative methods, multilevel methods, and preconditioned projection methods, respectively. The results can be extended to DTMCs based on Kronecker products with minor modifications. Areas that need further research are mentioned as they are discussed.

Our contribution to this area over the years corresponds to work along iterative methods based on splittings and their block versions [11], associated preconditioners to be used with projection methods [4], near complete decomposability [8], a method based on iterative disaggregation for a class of lumpable MCs [9], a class of multilevel methods [3], and a recent method based on decomposition for weakly interacting subsystems [1].

Keywords-Markov chains; Kronecker products; reordering; grouping; lumping; block iterative methods; multilevel methods; preconditioned projection methods;

\section{ACKNOWLEDGMENT}

The author gratefully acknowledges grant TÜBA-GEBIP of the Turkish Academy of Sciences.

\section{REFERENCES}

[1] Y. Bao, I. N. Bozkurt, T. Dayar, X. Sun, And K. S. TRIVEDI, Decompositional analysis of Kronecker structured Markov chains, to appear in Electronic Transactions on Numerical Analysis.

[2] P. BuCHHOLZ, Structured analysis approaches for large Markov chains, Applied Numerical Mathematics, 31 (1999), pp. 375404.

[3] P. BuchHolz AND T. DAYAR, Comparison of multilevel methods for Kronecker structured Markovian representations, Computing, 73 (2004), pp. 349-371.

[4] P. BUCHHOLZ AND T. DAYAR, Block SOR preconditioned projection methods for Kronecker structured Markovian representations, SIAM Journal on Scientific Computing, 26 (2005), pp. 1289-1313.

[5] P. BuChHOlZ AND P. KeMPER, Kronecker based representations of large Markov chains, in Validation of Stochastic Systems, Lecture Notes in Computer Science 2925, B. Haverkort, H. Hermanns, and M. Siegle, eds., Springer Verlag, 2004, pp. 256-295.

[6] M. DAVIO, Kronecker products and shuffle algebra, IEEE Transactions on Computers, C-30 (1981), pp. 116-125.

[7] T. DAYAR, Analyzing Markov chains based on Kronecker products, in MAM 2006: Markov Anniversary Meeting, A. N. Langville and W. J. Stewart, eds., Boson Books, Raleigh, North Carolina, USA, 2006, pp. 279-300.

[8] O. Gusak, T. DAYAR, AND J.-M. Fourneau, Stochastic automata networks and near complete decomposability, SIAM Journal on Matrix Analysis and Applications, 23 (2001), pp. $581-599$

[9] O. GusaK, T. DAYAR, AND J.-M. Fourneau, Lumpable continuous-time stochastic automata networks, European Journal of Operational Research, 148 (2003), pp. 436-451.

[10] W. J. Stewart, K. ATIF, AND B. PlateAu, The numerical solution of stochastic automata networks, European Journal of Operational Research, 86 (1995), pp. 503-525.

[11] E. UYSAL AND T. DAYAR, Iterative methods based on splittings for stochastic automata networks, European Journal of Operational Research, 110 (1998), pp. 166-186. 